\title{
Complement in ANCA-Associated Vasculitis
}

\author{
J Charles Jennette, MD, Hong Xiao, MD, and Peiqi Hu, MD \\ Department of Pathology and Laboratory Medicine, and UNC Kidney Center University of North \\ Carolina, Chapel Hill, NC
}

\section{Abstract}

Antineutrophil cytoplasmic autoantibodies (ANCA) are the likely cause for necrotizing small vessel vasculitis and crescentic glomerulonephritis. Unlike other forms of crescentic glomerulonephritis induced by immune complexes or anti-glomerular basement membrane (antiGBM) antibodies that have conspicuous vessel wall immunoglobulin and complement, there is a paucity, although usually not an absence, of vessel wall immunoglobulin and complement in ANCA-associated glomerulonephritis. In spite of this comparatively lower level and more localized distribution of vessel wall complement, experimental and clinical observations strongly incriminate alternative complement pathway activation as critically important in the pathogenesis of ANCA disease. Experimental data in animal models and in vitro experiments demonstrate that primed neutrophils are activated by ANCA, which generates C5a that engages C5a receptors on neutrophils. This attracts and in turn primes more neutrophils for activation by ANCA. In patients with ANCA disease, plasma levels of C3a, C5a, soluble C5b-9 and Bb have been reported to be higher in active disease than in remission, whereas no difference was reported in plasma $\mathrm{C} 4 \mathrm{~d}$ in active versus remission ANCA disease. Thus, experimental and clinical data support the hypothesis that ANCA-induced neutrophil activation activates the alternative complement pathway and generates C5a. C5a not only recruits additional neutrophils through chemotaxis but also primes neutrophils for activation by ANCA. This creates a self-fueling inflammatory amplification loop that results in the extremely destructive necrotizing vascular injury.

\section{INTRODUCTION}

Antineutrophil cytoplasmic autoantibodies (ANCA) are associated with and are the likely cause for a distinctive form of systemic necrotizing small vessel vasculitis and necrotizing crescentic glomerulonephritis (NCGN) with a paucity of glomerular immunoglobulin deposits $(1,2)$. ANCA disease has many different clinicopathologic phenotypes, including renal limited pauci-immune NCGN, microscopic polyangiitis (MPA) with systemic small vessel vasculitis but no granulomatosis or asthma, granulomatosis with polyangiitis (Wegener's) (GPA) with small vessel vasculitis and granulomatosis, and eosinophilic

\footnotetext{
(C) 2013 Elsevier Inc. All rights reserved.
}

Corresponding Author: J. Charles Jennette, MD, Brinkhous Distinguished Professor and Chair, Department of Pathology and Laboratory Medicine, University of North Carolina at Chapel Hill, Chapel Hill, NC 27599, Phone: 919-966-4676, jcj@ med.unc.edu.

Publisher's Disclaimer: This is a PDF file of an unedited manuscript that has been accepted for publication. As a service to our customers we are providing this early version of the manuscript. The manuscript will undergo copyediting, typesetting, and review of the resulting proof before it is published in its final citable form. Please note that during the production process errors may be discovered which could affect the content, and all legal disclaimers that apply to the journal pertain. 
granulomatosis with polyangiitis (Churg-Strauss) (EGPA) with small vessel vasculitis, granulomatosis and asthma (3). The vasculitis and glomerulonephritis in pauci-immune NCGN, MPA, GPA and EGPA are histologically indistinguishable and are characterized in the acute phase by necrosis with lysis of vessel walls and adjacent tissue. By immunofluorescence microscopy, complement components are a conspicuous component of the immune deposits in the walls of vessels affected by immune complex mediated and antiglomerular basement membrane antibody (anti-GBM) mediated glomerulonephritis and vasculitis; and substantial evidence supports a pathogenic role for complement in these diseases. However, ANCA NCGN and ANCA vasculitis have comparatively much less complement detected by immunofluorescence microscopy in involved vessels, although localized accumulation of complement components are frequently identified at sites of acute vascular inflammation and necrosis. Because of this relative paucity of complement in vessels, complement was not initially envisioned as an important participant in the pathogenesis of ANCA vasculitis and ANCA GN. However, alternative complement pathway activation is now recognized as a likely pivotal mediator in the induction of ANCA-induced acute vascular inflammation because of mounting evidence from experimental animal models, in vitro experiments, and clinical observations (2).

\section{PATHOLOGIC FEATURES OF AAV}

Any proposed pathogenic mechanism for ANCA disease must be in accord with the pathologic features of the observed acute vascular inflammation. Based on observations in animal models of ANCA disease as well as examination of biopsy specimens from patients, the initial inflammatory and necrotizing injury in ANCA vasculitis is characterized histologically by segmental lytic necrosis with admixed and adjacent neutrophils that are undergoing karyorrhexis (leukocytoclasia) (Figure 1a) $(2,4,5)$. Monocyte and macrophages become the predominant lesional leukocytes within a day or two. Conceptually, both neutrophils and monocytes enter the acute lesions, with neutrophils quickly undergoing disruption as evidenced by the leukocytoclasia, and with monocytes maturing into macrophages and persisting. Fibrinoid necrosis (Figure 1) is an almost constant finding at sites of acute injury and is caused by the activation of coagulation factors that spill from the plasma into necrotic vessel walls and perivascular tissue, where the coagulation cascade is activated by thrombogenic materials, including tissue factor, resulting in fibrin formation. Within a week, macrophages become the predominant cell type in lesions and the fibrinoid necrosis is replaced by collagenous matrix. With further chronicity, T lymphocytes are present along with the macrophages in the injured glomeruli.

In a study by Weidner et al. of 65 renal biopsy specimens obtained from patients with newly diagnosed ANCA NCGN, neutrophils and monocytes/macrophages were the dominant infiltrating cells in glomeruli, with 4.7 \pm 11.1 monocytes/macrophages, $3.2 \pm 7.4$ neutrophils and $1.3 \pm \mathrm{T}$ lymphocytes per glomerular cross section (6). Glomerular infiltration of CD15positive neutrophils was associated with acute glomerular lesions and CD68-positive monocytes/macrophages were associated with foci of glomerular necrosis and crescent formation. Glomerular CD3-positive lymphocytes were rare. Lymphocytes were most numerous in the interstitium adjacent to glomeruli with sclerosis or crescent formation. Another study by Cunningham et al. of ANCA glomerulonephritis that included acute and 
chronic glomerular lesions detected $7.3 \pm 6.1$ macrophages, $3.7 \pm 2.5$ lymphocytes and 2.8 \pm 1.7 neutrophils per glomerular cross section (7). This detection of $\mathrm{T}$ lymphocytes and macrophages in renal biopsy specimens, along with the paucity of immunoglobulin in the injured glomeruli, has prompted some investigators to propose that the glomerular injury is caused by a $\mathrm{T}$ cell-mediated immune response rather than an antibody-mediated mechanism (7). However, in an experimental murine model, acute pauci-immune NCGN can be induced by intravenous injection of anti-MPO IgG not only in wild type mice but also in Rag2-/mice that have no functioning T cells (8). Thus, anti-MPO IgG alone in the absence of functional $\mathrm{T}$ cells was fully capable of causing disease. In this murine model, neutrophils are essential effector cells because disease induction by anti-MPO $\operatorname{IgG}$ is completely prevented by depletion of circulating neutrophils (9). Also in this model, neutrophils predominate in the earliest lesions (e.g. 1 day after injection of anti-MPO $\operatorname{IgG}$ ) although by day 6 monocytes/macrophages have joined the neutrophils in the acute lesions (10).

Undoubtedly, T cells are critically important in the overall pathogenesis of ANCA disease in addition to neutrophils, monocytes and antibodies/B cells (11). For example, T cells are involved in the genesis of the pathogenic autoimmune response both through MHC mediated recognition of ANCA autoantigens and defective regulatory $\mathrm{T}$ cells that do not adequately suppress the ANCA autoimmune response. Further, T cells are involved in the orchestration of the responses to acute injury, including progression to fibrosis and the induction of granulomatous inflammation.

Although most investigators in the field currently conclude that the vasculitis and glomerulonephritis of ANCA disease are mediated by the autoantibodies, the basis for this was not clear when ANCA were first discovered because most ANCA associated glomerulonephritis is characterized immunohistologically by a paucity of immunoglobulin and complement deposition in vessel walls and glomeruli, which differs from immune complex mediated and anti-GBM glomerulonephritis $(3,4)$. However, a paucity is not an absence, and many patients with ANCA NCGN have evidence for at least low levels of immunoglobulin and complement components in glomeruli. For example, in a study by Haas and Eustace of 126 renal biopsies with ANCA-associate or arteritis-associated NCGN $15 \%$ had positive immunofluorescence microscopy findings for $\operatorname{IgG}, 49 \%$ for $\operatorname{IgM}, 57 \%$ for $\mathrm{C} 3$ and $8 \%$ for $\mathrm{C} 1 \mathrm{q}(12)$. When positive, the staining was mild in most instances ( $\mathcal{Q}+$ on a 0 to $4+$ scale). Glomerular electron dense deposits were identified in $54 \%$ of these biopsies, although these were usually sparse. Given that the complement deposition was more frequent than the immunoglobulin deposition, some of the electron dense deposits may contain only complement. Electron dense deposits can result not only from immune complex deposition but also from deposition of complement components resulting from complement activation in the absence of antibodies, for example in $\mathrm{C} 3$ glomerulopathy caused by dysregulated alternative pathway complement activation (13) (see Chapter 2 Barbour et al).

In summary, based on pathologic observations in biopsies from patients and in animal models, the initial acute vascular lesions of ANCA disease, including glomerulonephritis, appear to begin with the accumulation of neutrophils rapidly followed by the influx of monocytes/macrophages and induction of vascular necrosis. In spite of the relative paucity of immunoglobulin and complement in the injured glomeruli and vessels, both experimental 
results and observations in patients support a pathogenic role for both immunoglobulin and complement in the mediation of this acute necrotizing injury.

\section{EXPERIMENTAL EVIDENCE FOR PATHOGENIC COMPLEMENT ACTIVATION IN ANCA DISEASE}

Numerous in vitro studies and multiple animal models substantiate a pathogenic role for ANCA (2). The evidence shows that cytokine-primed neutrophils display ANCA-antigens (MPO and PR3) at the cell surface where interaction with ANCA causes neutrophil activation by both $\mathrm{Fc}$ receptor engagement and by $\mathrm{F}(\mathrm{ab}$ ') 2 binding to antigen on the neutrophil surface. ANCA-activated neutrophils adhere to and penetrate vessel walls, and release destructive enzymes and oxygen radicals that cause vascular necrosis.

The initial evidence that complement activation played an important role in the pathogenesis of ANCA disease came from animal model studies. Xiao et al. investigated the effect of depletion or blockade of complement activation on the induction of NCGN in mice by intravenous injection of anti-MPO-IgG (14). The effect of complement depletion by a single intraperitoneal injection of cobra venom factor (CVF) 4 hours prior to injection of nephritogenic anti-MPO IgG was assessed. When the mice were sacrificed 6 days later, all mice that did not have CVF complement depletion developed albuminuria, leukocyturia, and hematuria whereas the mice that received CVF did not. Pathologic examination revealed that all mice that did not receive CVF developed NCGN with neutrophil and macrophage infiltration and low-level glomerular IgG and $\mathrm{C} 3$ deposition, whereas the mice that received CVF developed no glomerular lesions. At the time of sacrifice, mice with and without CVF had the same levels of circulating anti-MPO.

To identify which complement activation pathway was required for anti-MPO mediated inflammation, anti-MPO IgG was administered to mice with knockout (KO) $\mathrm{C}$, which is required for all pathways of complement activation, $\mathrm{C} 4$, which is required for classic and lectin binding pathway activation, or factor $\mathrm{B}$, which is required for alternative pathway activation (Figure 2). All control wild type mice given anti-MPO developed NCGN. C5 KO mice developed no NCGN, confirming the results of the CVF experiment. All C4 KO mice developed NCGN comparable to wild type mice, indicating that neither the classic nor lectin complement activation pathways are required for disease induction. In contrast, none of the factor B KO mice developed NCGN, indicating that alternative pathway complement activation is required for induction of NCGN by anti-MPO IgG.

To identify a mechanism by which complement activation can occur in ANCA disease, IgG was isolated from patients with MPO-ANCA or PR3-ANCA, and incubated with cytokineprimed normal human neutrophils (14)., ANCA IgG is known to cause ANCA-induced neutrophil activation in vitro (15). The supernatants from these incubations were added to normal human serum from the donor of the neutrophils. Complement activation was measured by C3a ELISA. MPO-ANCA IgG and PR3-ANCA IgG, but not control IgG, induced neutrophils to release factors that caused complement activation with generation of C3a (14). This supports the hypothesis that activation of neutrophils by ANCA IgG results in the release of factors (as yet not identified but possibly properdin or factor B) that cause 
complement activation, which in turn amplifies the recruitment, priming and activation of more neutrophils.

Huugen et al. used a similar mouse model to demonstrate the importance of $\mathrm{C} 5$ activation in MPO-ANCA mediated NCGN (16). Mice received a C5-inhibiting monoclonal antibody (anti-C5) or diluent alone $8 \mathrm{~h}$ before or 1 day after intravenous injection of a nephritogenic dose of anti-MPO IgG and lipopolysaccharide (LPS). LPS was administered because it is known to enhance the severity of anti-MPO induced NCGN (10). All mice that received anti-MPO but no anti-C5 developed nephritic urinary findings and NCGN by pathologic examination at day 7. Mice that were pretreated with anti-C5 developed no nephritic urinary findings and no NCGN at day 7. Pretreatment with anti-C5 also reduced glomerular neutrophil influx at day 1. Administration of anti-C5 1 day after administration of anti-MPO resulted in a marked diminution of urinary abnormalities and $>80 \%$ reduction in glomerular crescents.

Schreiber et al. used another anti-MPO induced NCGN mouse model to confirm the importance of $\mathrm{C} 5$ activation and to demonstrate $\mathrm{C} 5 \mathrm{a}$ receptor (C5aR) engagement as a critical step in the amplification of MPO ANCA-induced injury (17). MPO KO mice were immunized with murine MPO, irradiated, and transplanted with bone marrow from wildtype mice or C5aR-deficient mice, which underwent engraftment and produced circulating MPO-positive neutrophils. Thus, after immunization and transplantation, these MPO KO mice had circulating anti-MPO and circulating neutrophils containing MPO. All (6 of 6) MPO KO mice that received wild-type marrow developed NCGN, whereas only 1 of 8 MPO KO mice that received C5aR-deficient marrow developed NCGN $(\mathrm{P}<0.05)$. Proteinuria and glomerular neutrophil influx also were significantly reduced in mice with C5aR-deficient neutrophils.

Schreiber et al. also showed that supernatants from human neutrophils activated by human ANCA IgG activate complement in normal serum producing C5a (17). This conditioned serum is able to prime neutrophils for activation by ANCA. This priming can be prevented by $\mathrm{C} 5 \mathrm{aR}$ blockade but not by $\mathrm{C} 3 \mathrm{aR}$ blockade (Figure 3), and can be replicated using recombinant $\mathrm{C} 5 \mathrm{a}$ but not $\mathrm{C} 3 \mathrm{a}$.

Additional studies by Hao et al. have confirmed the ability of C5a to prime neutrophils for enhanced activation by ANCA, and have shown that activation of p38 mitogen activated protein kinase (p38MAPK), extracellular signal-regulated kinase (ERK) and phosphoinositol 3- kinase (PI3 K) are involved in C5a induced translocation of ANCA antigens to the surface where they can interact with ANCA resulting in neutrophil activation $(18,19)$. In addition, inhibiting protein kinase $\mathrm{C}$ (PKC) pathway with bisindolylmaleimide (BIS) inhibits C5a induced neutrophil priming (19).

The importance of C5aR also has been confirmed by Xiao et al. in the mouse model of ANCA GN caused by injection of anti-MPO IgG (20). Wild type mice, C5aR KO mice, and C6 KO mice were injected intravenously with mouse anti-mouse MPO IgG and sacrificed on day 6. All WT mice developed NCGN, whereas only 1 of 6 C5aR KO developed NCGN, confirming the pathogenic importance of C5aR. Further, all 5 C6 KO mice developed 
NCGN with similar severity to WT mice indicating that C6 (and by implication the membrane attack complex) is not required for mediation of anti-MPO induced NCGN.

These observations suggest that blockade of the C5aR might have therapeutic benefit. To test this hypothesis, the effect of a small molecule antagonist of human C5aR on induction of NCGN by anti-MPO IgG was tested in mice with knocked in human C5aR and knocked out mouse C5aR (20). This small molecule antagonist of human C5aR showed a dose dependent reduction in anti-MPO induced NCGN, further confirming the importance of C5aR engagement in pathogenesis (20). A Phase 2 trial is underway in Europe to test the effectiveness of this C5aR antagonist in patients with ANCA NCGN (EU Clinical Trials Register ID: EUCTR2011-001222-15-GB).

Thus, multiple lines of experimental data in animal models and in vitro experiments using human neutrophils and patient ANCA support the hypothesis that an important event in ANCA mediated glomerulonephritis is alternative complement pathway activation by ANCA-activated neutrophils, which generates C5a that engages C5a receptors on neutrophils, which in turn primes the neutrophils for activation by ANCA (Figure 4). In addition, C5a is a potent chemoattractant for neutrophils (21). Thus, C5a not only primes neutrophils in the inflammatory microenvironment for activation by ANCA, but also attracts additional neutrophils into the site of inflammation.

\section{EVIDENCE FOR PATHOGENIC COMPLEMENT ACTIVATION IN ANCA DISEASE PATIENTS}

A role for complement activation in the pathogenesis of ANCA disease is supported by the experimental evidence from in vitro studies demonstrating that patient MPO-ANCA and PR3-ANCA IgG can activate neutrophils to release factors that in turn activate complement, and by experimental animal studies that clearly show an important role for alternative complement pathway activation in models of MPO-ANCA disease. The absence of overt depression of serum $\mathrm{C} 3$ and $\mathrm{C} 4$ in ANCA disease patients, along with the relative paucity of glomerular staining for $\mathrm{C} 3$ and $\mathrm{C} 4$, discouraged considering complement activation as an important mediator of ANCA disease. However, there is mounting evidence from observations of ANCA disease patients that supports the pathogenic importance of complement activation, although most of these observations in patients have not yet been substantiated by confirmatory studies.

Important observations in ANCA disease patients that support a role for alternative complement pathway activation have been reported by Ming-Hui Zhao and his associates $(22,23)$. They measured the plasma complement activation profile in 66 patients with active ANCA disease and compared this to the profile in 54 patients with ANCA disease in remission. Plasma levels of $\mathrm{C} 3 \mathrm{a}, \mathrm{C} 5 \mathrm{a}$, soluble $\mathrm{C} 5 \mathrm{~b}-9$ and $\mathrm{Bb}$ were higher in active disease than in remission, whereas levels of properdin were significantly lower in active disease than in remission (Figure 5). There was no significant difference in plasma C4d in ANCA disease versus remission. In addition, the plasma level of $\mathrm{Bb}$ in patients with active ANCA disease correlated with the \% cellular crescents in renal biopsies, erythrocyte sedimentation 
rate, and Birmingham Vasculitis Activity Scores (22). These finding support alternative pathway and not classic or lectin pathway activation.

Immunohistochemical examination of renal biopsy specimens from ANCA disease patients revealed $\mathrm{Bb}, \mathrm{C} 3 \mathrm{~d}$, and C5b-9 in glomeruli and in small arteries (23). This confirmed earlier findings reported by the same group in seven patients with MPO-ANCA NCGN that showed deposits of C5b-9, C3d, factor B and factor P in glomeruli and small blood vessels with active vasculitis. Mannose binding lectin (MBL) and C4d were not detected. These observations support a role for alternative complement pathway activation but not classic or lectin pathways in the pathogenesis of ANCA induced inflammation.

The preferential focal accumulation of complement activation components at sites of glomerular and vascular inflammation and necrosis $(22,23)$ (Figure 1c) is very different from the more evenly distributed immunoglobulin and complement components seen in affected vessels in immune complex and anti-GBM mediated inflammation. In all forms of immune complex and anti-GBM glomerulonephritis, virtually every segment of every glomerulus has immune deposits by immunofluorescence microscopy, which is consistent with widespread localization of immune complex and anti-GBM deposits in vessel walls as an initial process that secondarily mediates the recruitment of inflammatory cells. In marked contrast, in ANCA glomerulonephritis, complement components and immunoglobulin are markedly accentuated at sites of inflammation and necrosis, and less apparent or absent in segments and glomeruli with no histopathologic changes (Figure 1c). This absence or paucity of complement and immunoglobulin in vessels without inflammation, and the accentuation of staining at sites of inflammation and necrosis, are consistent with a more interdependent involvement of complement activation with induction of inflammation primarily in the microenvironment at localized sites of inflammation and necrosis in ANCA disease.

\section{SUMMARY}

Thus, multiple lines of experimental data in animal models, in vitro experiments using human neutrophils and patient ANCA, and observations in patients with ANCA disease all support the hypothesis that an important event in ANCA mediated glomerulonephritis and vasculitis is alternative complement pathway activation by ANCA-activated neutrophils, which generates $\mathrm{C} 5 \mathrm{a}$ that engages $\mathrm{C} 5 \mathrm{a}$ receptors on neutrophils, which in turn primes the neutrophils for activation by ANCA (Figure 2). In addition, C5a is a potent chemoattractant for neutrophils (21). Thus, C5a complements the inflammation not only by priming neutrophils in the inflammatory microenvironment for activation by ANCA, but also by attracting more neutrophils into the site of inflammation. This creates an especially toxic self-perpetuating inflammatory amplification loop that results in the extremely destructive necrotizing injury of ANCA disease.

\section{REFERENCES}

1. Falk RJ, Jennette JC. ANCA Disease: Where Is This Field Going? J Am Soc Nephrol. 2010; 21:745-752. [PubMed: 20395376] 
2. Jennette JC, Falk RJ, Hu P, Xiao H. Pathogenesis of Anti-neutrophil Cytoplasmic Autoantibody Associated Small Vessel Vasculitis. Annu Rev Pathol. 2013; 8:139-160. [PubMed: 23347350]

3. Jennette JC, Falk RJ, Bacon PA, Basu N, Cid MC, Ferrario F, Flores-Suarez LF, Gross WL, Guillevin L, Hagen EC, Hoffman GS, Jayne DR, Kallenberg CGM, Lamprecht P, Langford CA, Luqmani RA, Mahr AD, Matteson EL, Merkel PA, Ozen S, Pusey CD, Rasmussen N, Rees AJ, Scott DGI, Specks U, Stone JH, Takahashi K, Watts RA. 2012 Revised International Chapel Hill Consensus Conference Nomenclature of Vasculitides. Arthritis Rheum. 2013; 65:1-11. [PubMed: 23045170]

4. Jennette JC, Falk RJ. The role of pathology in the diagnosis of systemic vasculitis. Clin Exp Rheumatol. 2007; 25(1 Suppl 44):52-62.

5. Jennette JC. Nomenclature and classification of vasculitis: lessons learned from granulomatosis with polyangiitis (Wegner's granulomatosis). Clin Exp Immunol. 2011; 164(Suppl 1):7-10. [PubMed: 21447122]

6. Weidner S, Carl M, Riess R, Rupprecht HD. Histologic analysis of renal leukocyte infiltration in antineutrophil cytoplasmic antibody-associated vasculitis: importance of monocyte and neutrophil infiltration in tissue damage. Arthritis Rheum. 2004; 50:3651-3657. [PubMed: 15529388]

7. Cunningham MA, Huang XR, Dowling JP, Tipping PG, Holdsworth SR. Prominence of cellmediated immunity effectors in "pauci-immune" glomerulonephritis. J Am Soc Nephrol. 1999; 10:499-506. [PubMed: 10073600]

8. Xiao H, Heeringa P, Hu P, Liu Z, Zhao M, Aratani Y, Maeda N, Falk RJ, Jennette JC. Antineutrophil cytoplasmic autoantibodies specific for myeloperoxidase cause glomerulonephritis and vasculitis in mice. J Clin Invest. 2002; 110:955-963. [PubMed: 12370273]

9. Xiao H, Heeringa P, Liu Z, Huugen D, Hu P, Falk RJ, Jennette JC. The role of neutrophils in the induction of glomerulonephritis by anti-myeloperoxidase antibodies. Am J Pathol. 2005; 167:3945. [PubMed: 15972950]

10. Huugen D, Xiao H, Esch A, Falk RJ, Peutz-Kootstra CJ, Buurman WA, Cohen Tervaert JW, Jennette JC, Heeringa P. Aggravation of anti-myeloperoxidase antibody induced glomerulonephritis by bacterial lipopolysaccharide: role of tumor necrosis factor a. Am J Pathol. 2005; 167:47-58. [PubMed: 15972951]

11. Wilde B, Thewissen M, Damoiseaux J, van Paassen P, Witzke O, Tervaert JW. T cells in ANCAassociated vasculitis: what can we learn from lesional versus circulating T cells? Arthritis Res Ther. 2010; 12:204. [PubMed: 20236453]

12. Haas M, Eustace JA. Immune complex deposits in ANCA-associated crescentic glomerulonephritis: A study of 126 cases. Kidney Int. 2004; 65:2145-2152. [PubMed: 15149327]

13. Fakhouri F, Frémeaux-Bacchi V, Noël LH, Cook HT, Pickering MC. C3 glomerulopathy: a new classification. Nat Rev Nephrol. 2010; 6:494-499. [PubMed: 20606628]

14. Xiao H, Schreiber A, Heeringa P, Falk RJ, Jennette JC. Alternative complement pathway in the pathogenesis of disease mediated by antineutrophil cytoplasmic autoantibodies. Am J Pathol. 2007; 170:52-64. [PubMed: 17200182]

15. Falk RJ, Terrell R, Charles LA, Jennette JC. Anti-neutrophil cytoplasmic autoantibodies induce neutrophils to degranulate and produce oxygen radicals. Proc Nat Acad Sci USA. 1990; 87:41154119. [PubMed: 2161532]

16. Huugen D, van Esch A, Xiao H, Peutz-Kootstra CJ, Buurman WA, Cohen Tervaert JW, Jennette JC, Peter Heeringa P. Inhibition of complement factor C5 protects against anti-myeloperoxidase antibody-mediated glomerulonephritis in mice. Kidney Int. 2007; 71:646-654. [PubMed: 17299525]

17. Schreiber A, Xiao H, Jennette JC, Schneider W, Luft FC, Kettritz R. C5a receptor mediates neutrophil activation and ANCA-induced glomerulonephritis. J Am Soc Nephrol. 2009; 20:289_ 298. [PubMed: 19073822]

18. Hao J, Meng LQ, Xu PC, Chen M, Zhao MH. p38MAPK, ERK and PI3K signaling pathways are involved in C5a-primed neutrophils for ANCA-mediated activation. PLoS One. 2012; 7:e38317. [PubMed: 22675451]

19. Hao J, Chen M, Zhao MH. Involvement of protein kinase $\mathrm{C}$ in C5a-primed neutrophils for ANCAmediated activation. Mol Immunol. 2013; 54:68-73. [PubMed: 23201854] 
20. Xiao H, Dairaghi DJ, Powers JP, Ertl LS, Baumgart T, Wang Y, Seitz LC, Penfold MET, Gao L, Hu1 P, Lu B, Gerard NP, Gerard C, Schall TJ, Jaen JC, Falk RJ, Jennette JC. C5a Receptor (CD88) Blockade Protects against MPO-ANCA Glomerulonephritis. J Am Soc Nephrol. 2013 submitted.

21. Chen M, Daha MR, Kallenberg CG. The complement system in systemic autoimmune disease. J Autoimmun. 2010; 34:J276-J286. [PubMed: 20005073]

22. Gou SJ, Yuan J, Chen M, Yu F, Zhao MH. Circulating complement activation in patients with antineutrophil cytoplasmic antibody-associated vasculitis. Kidney Int. 2012; 83:129-137. [PubMed: 22913983]

23. Xing GQ, Chen M, Liu G, Heeringa P, Zhang JJ, Zheng XEJ, Kallenberg CG, Zhao MH. Complement activation is involved in renal damage in human antineutrophil cytoplasmic autoantibody associated pauci-immune vasculitis. J Clin Immunol. 2009; 29:282-291. [PubMed: 19067130]

24. Gigante A, Salviani C, Giannakakis K, Rosato E, Barbano B, Moroso A, Gasperini ML, Nofroni I, Salsano F, Cianci R, Pugliese F. Clinical and histological outcome predictors in renal limited pauci-immune crescentic glomerulonephritis: a single centre experience. Int J Immunopathol Pharmacol. 2012; 25:287-292. [PubMed: 22507343] 

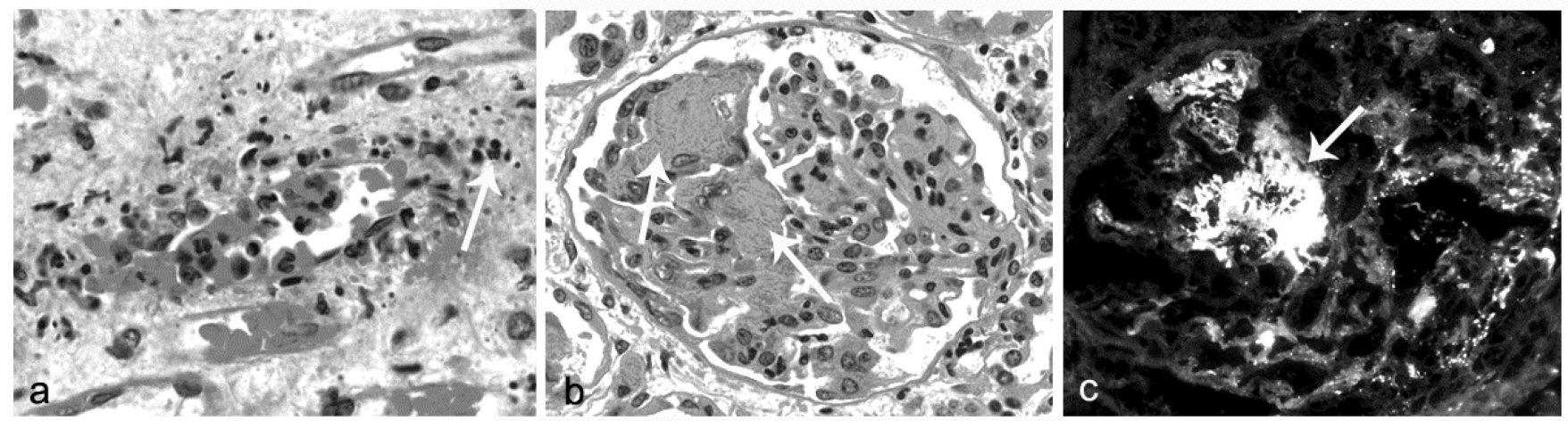

Figure 1.

Panel a: Early acute medullary angiitis in a patient with ANCA disease showing margination and diapedesis of neutrophils and adjacent leukocytoclasia (white arrow, H\&E stain). Panel b: Early acute necrotizing ANCA glomerulonephritis with a slight increase in glomerular neutrophils and segmental fibrinoid necrosis (white arrows, H\&E stain). Panel c: Early acute necrotizing ANCA glomerulonephritis with segmental staining for $\mathrm{C} 3$ by immunofluorescence microscopy corresponding to segmental fibrinoid necrosis (FITC antiC3). 


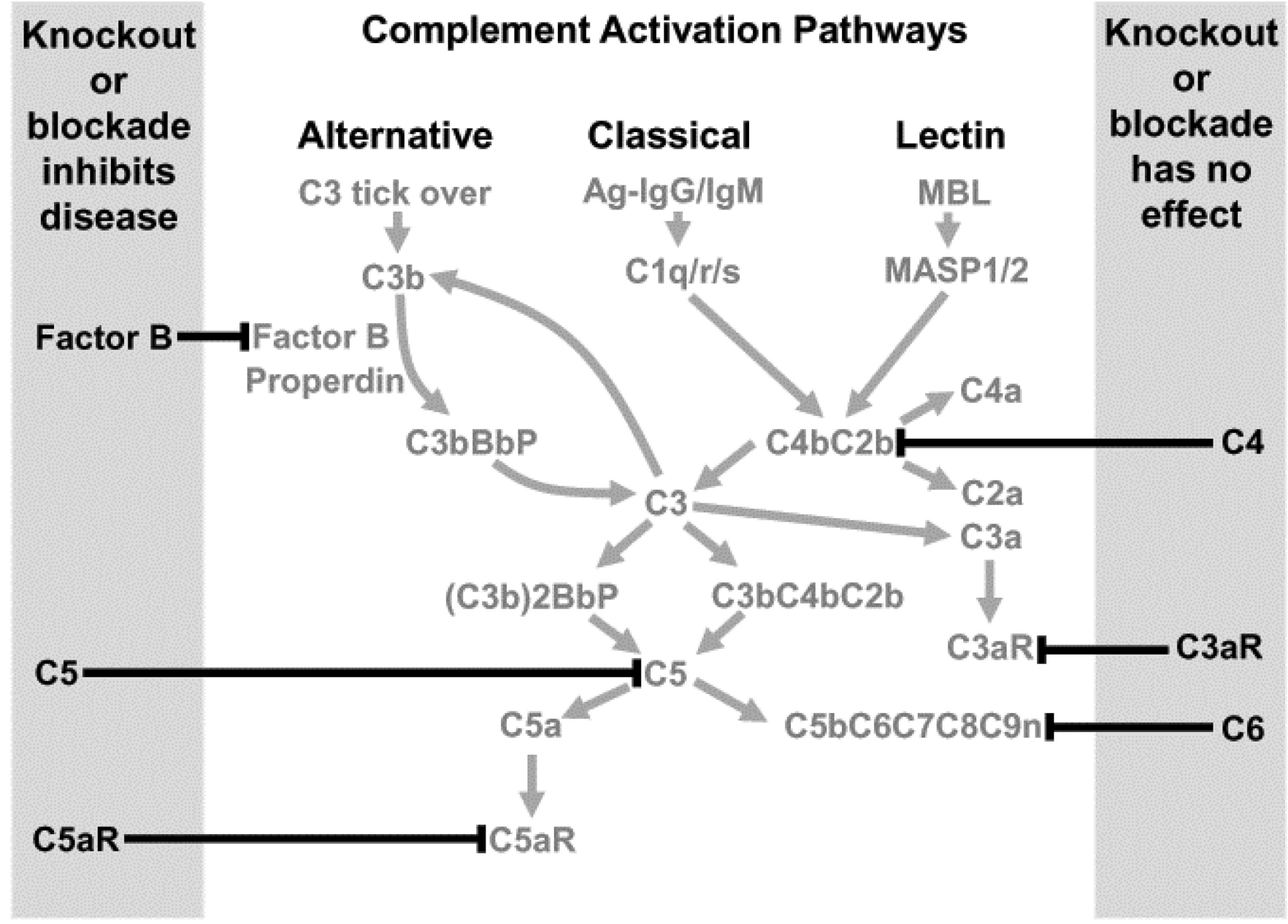

Figure 2.

Diagram showing the effects of knockout or blockade of specific elements of the alternative, classical and lectin pathways of complement activation on the pathogenesis of anti-MPO induced murine crescentic glomerulonephritis. Note that disruption of the alternative pathway, and blockade of $\mathrm{C} 5$ and $\mathrm{C} 5 \mathrm{aR}$ inhibits disease; but not disruption of the classical or lectin pathways, depletion of $\mathrm{C} 6$ or blockade of $\mathrm{C} 3 \mathrm{aR}$ (shown only in vitro). This figure is based on data reported in references 14, 16, 17 and 20. 

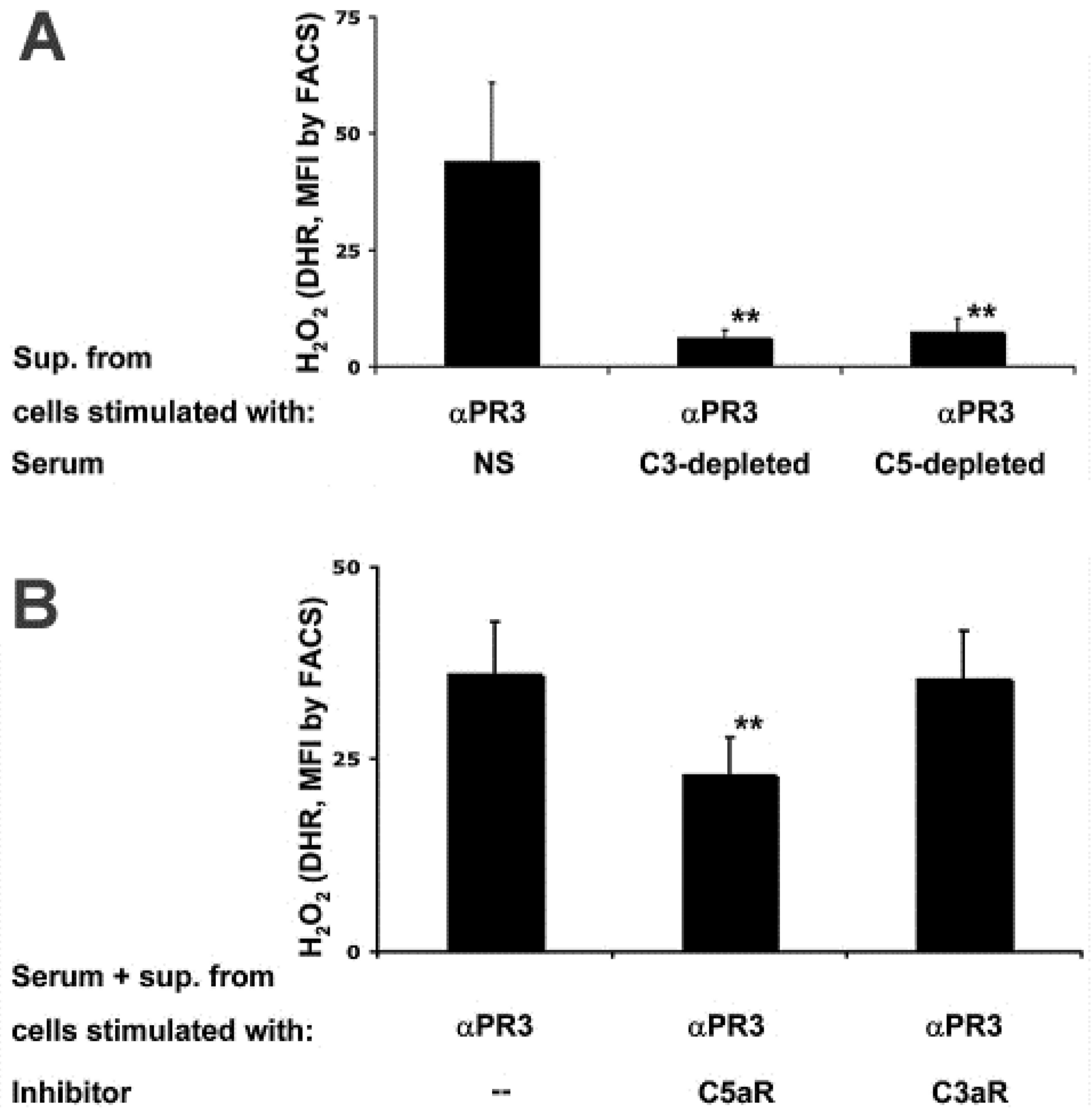

Serum + sup. from

Figure 3.

A: TNF-primed neutrophils were stimulated with monoclonal anti-PR3 and their supernatants (conditioned serum) were incubated with normal serum (NS), C3-depleted serum, or C5-depleted serum. These conditioned sera were then used to prime normal neutrophils for activation by anti-PR3 with resultant respiratory burst. Depletion of C3 or C5 from serum prevented the generation of ANCA-induced priming factor(s). B: Normal neutrophils were preincubated with a blocking antibody to $\mathrm{C} 5 \mathrm{aR}$ or a $\mathrm{C} 3 \mathrm{aR}$ antagonist, 
followed by priming with conditioned serum and subsequently activated with anti-PR3. Anti-PR3 induced respiratory burst was inhibited by blocking C5aR but not C3aR. 


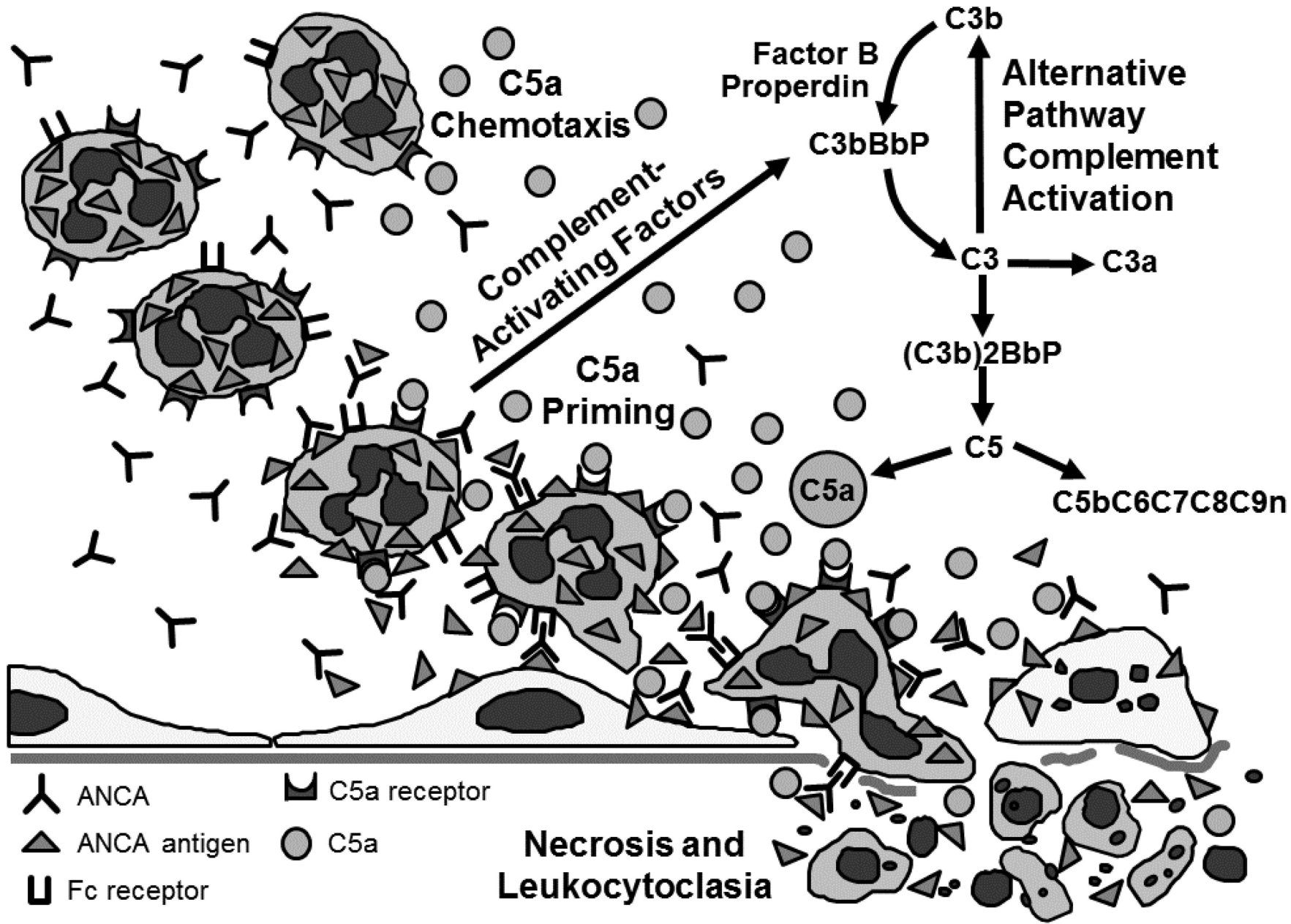

Figure 4.

Diagram depicting C5a as a prime mover at the center of an inflammatory amplification loop that mediates ANCA-induced vascular inflammation and nercrosis. Neutrophils activated by ANCA release factors that activate the alternative complement pathway, generating C5a. C5a is chemoattractant for neutrophils and primes neutrophils by C5aR engagement to display ANCA-antigens at the cell surface, which allows neutrophil activation by ANCA through FcR and Fab'2 binding. ANCA-activated neutrophils cause vascular necrosis and undergo leukocytoclasia. 
a
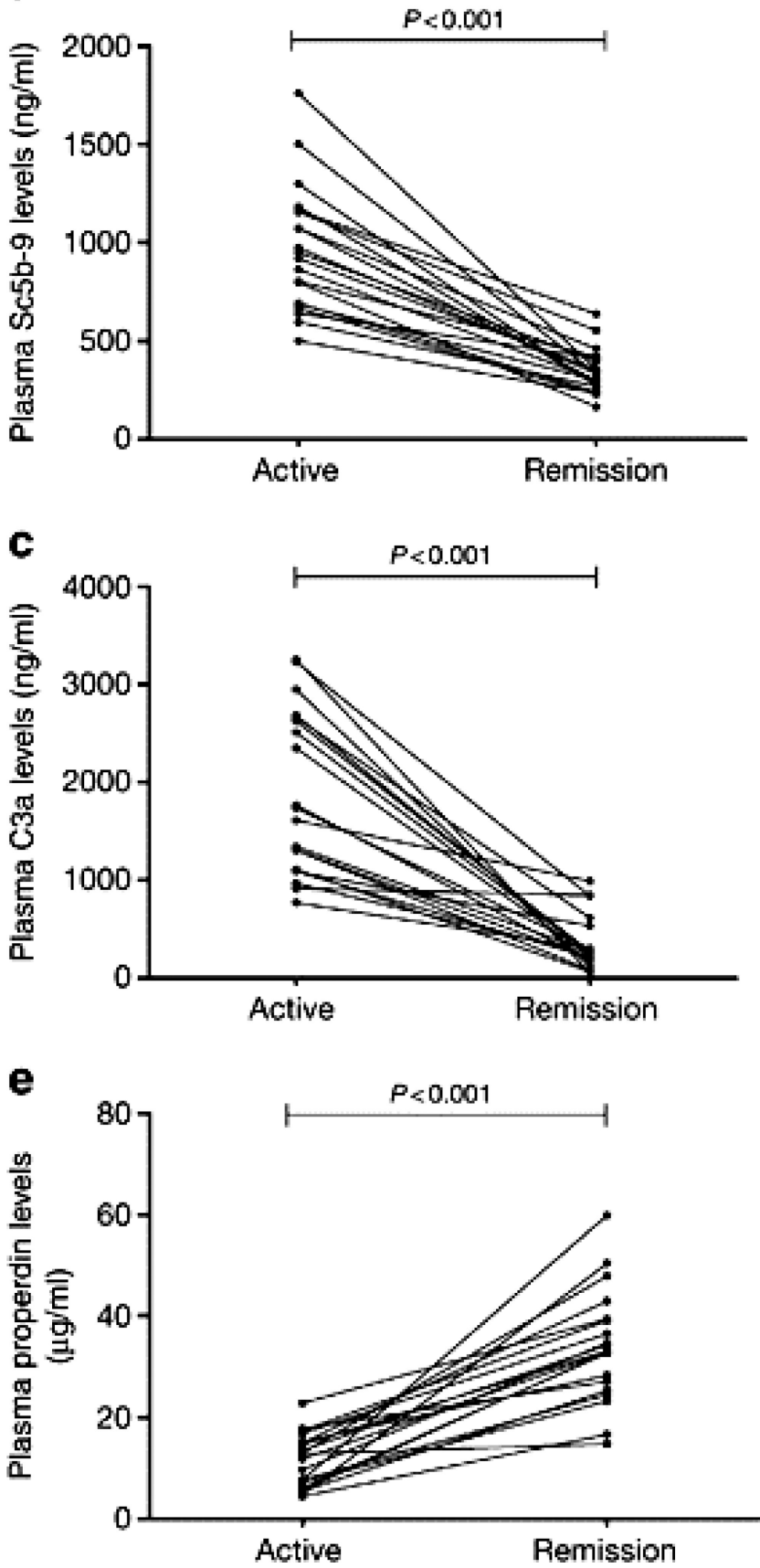

b
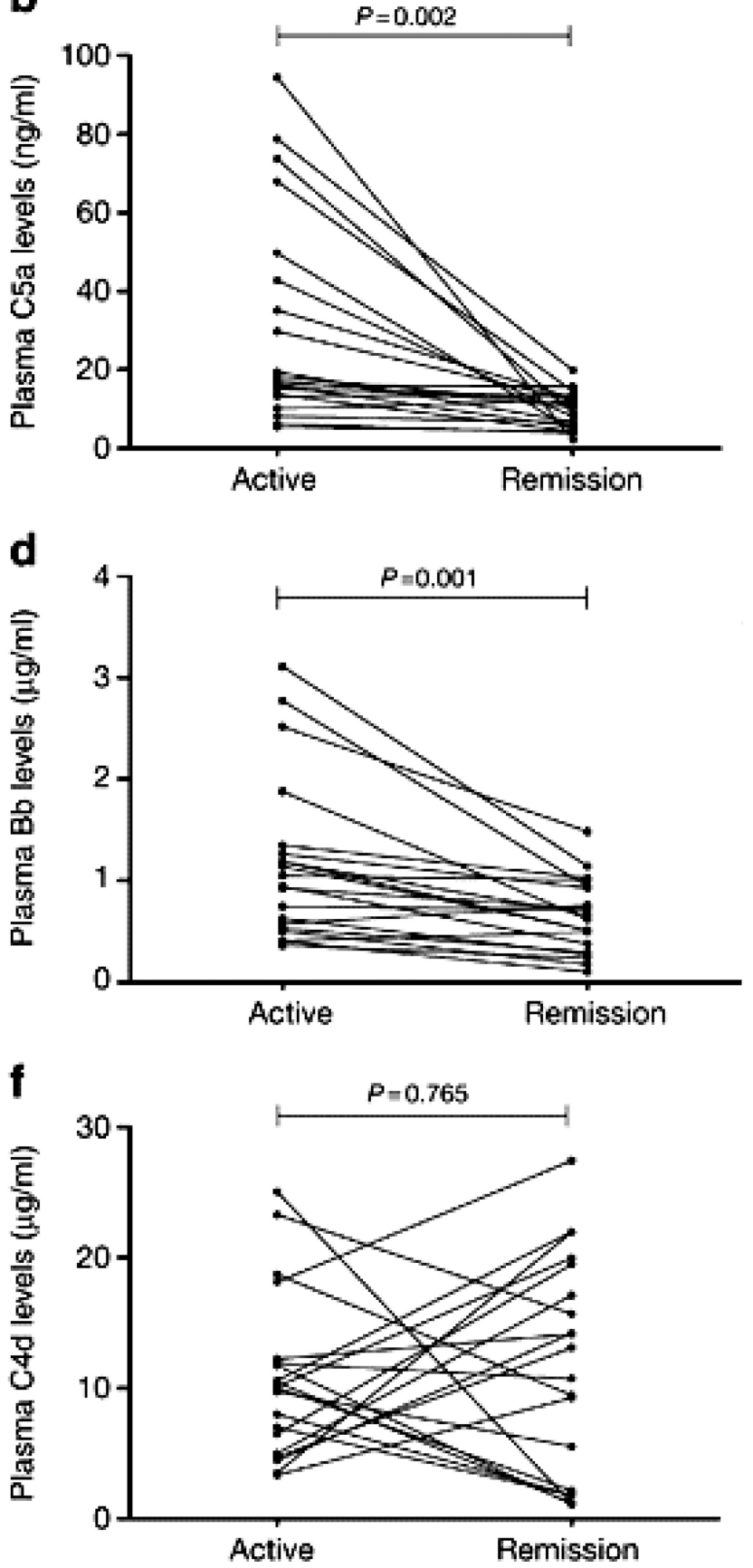

Figure 5.

Plasma complement levels in 20 ANCA disease patients during active disease compared to remission. (a) Plasma C5b-9. (b) Plasma C5a. (c) Plasma C3a. (d) Plasma Bb. (e) Plasma properdin. (f) Plasma C4d. Plasma levels of C3a, C5a, C5b-9, and Bb were significantly higher in active disease than remission, whereas plasma properdin were significantly lower in active disease. These findings are consistent with alternative pathway complement activation during active ANCA disease. (Reprinted from Reference 22 with permission) 